\title{
Enhancing understanding of analytic geometry by augmented reality
}

\section{Xenia-Rosemarie Reit}

Institute for mathematics education, Goethe-University Frankfurt, Germany.

\begin{abstract}
An augmented reality (AR) system is a technology which combines computergenerated representations or information and reality in real-time. Instead of substituting the real situation, as it is possible with dynamic geometry systems (DGS), AR adds virtual objects or information to reality to make the situation experiential. The project MalAR aims at investigating the impact of an augmented reality (AR) learning environment in the subject area of analytic geometry. The focus of the AR learning environment is an AR-App, which supports learners understanding of mathematics situations usually given through textbook tasks. Mathematics situations are implemented in an AR-App such that the situation gets visually and enactively explorable by changing perspectives using the own body movement. The added value of integrating AR in mathematics classes is worked out and based on different learning theories like embodied cognition. The AR-App and the project will be presented. With the findings and results of the study evidence-based didactical insights in the teaching and learning with $A R$ in mathematics instruction may be identified and needs for future AR-supported learning scenarios can be revealed.
\end{abstract}

Keywords: augmented reality; digital tools; mathematics education. 


\section{Introduction}

Increasing digitization not only modifies professional and private demands but also creates possibilities with high potential for teaching and learning. In order to integrate promising developments in schools, there is a need for teaching and learning concepts and teacher qualification. Making innovations available for teaching and learning, beyond the provision of hardware and software, is central.

In the field of digital media, the augmented reality technology (AR) gets great attention for a couple of years, although Sutherland already developed the first user interface in the early 60 's. Technological development of smartphones and their cameras made it interesting for educational research more recently. Not least the 2017's release of Apples AR Kit opened the market for public app-development in that field.

AR technology allows for completing the real word (e.g. the view through the smartphone camera) by virtual objects, material or information. The coexistence of virtual objects and real environment makes it possible to picture complex spatial relations in a so far unprecedented way (Avanitis, Petrou, Knight, Savas, \& Gargalakos, 2007). The experience of complex phenomena and the interaction with two- and three-dimensional objects by AR has never been more tangible (Kerawalla, Luckin, Seljeflot, \& Woolard, 2006; Klopfer \& Squire, 2008). Those advantages brought researcher to call AR an interdisciplinary key technology for the next years (Johnson, Levine, Smith, \& Haywood, 2010a, 2010b; Martin, et al., 2011).

However, the AR research in the field of education is still at an early stage (Wu, Lee, Chang, \& Jyh-Chong, 2013, p. 47). Especially in mathematics education there is a lack of studies investigating the use, advantages and key success factors of AR in teaching and learning. Kaufmann and Schmalstieg for example investigated the effects of AR on the concept of space (Kaufmann \& Schmalstieg 2003; Kaufmann, Steinbügl, Dünser, \& Glück, 2005). To exploit the full potential of AR, its additional value should be in focus. Based on this, the need for concrete learning environments can be worked out to provide an enriching access to AR for teachers and to support learners throughout the AR learning environment.

\section{Augmented reality (AR)}

An augmented reality (AR) system is a technology which combines computer-generated representations and reality in real-time (Milgram \& Kishino, 1994). Instead of substituting the real situation, as it is possible with dynamic geometry systems (DGS), AR adds objects or information to make it experiential (Azuma, 1997; Martin-Gutierrez, Saorin, Contero, Alcaniz, \& Perez-Lopez, 2010). 
Figure 1 shows the visualisation of a textbook task using the so far unpublished AR-App "GeometAR". A cube, a plane and a straight line, positioned within a fixed coordinate system, is added to the real world-view through the iPad-camera. The user can experience the augmented situation instead of only reading a textbook task by moving around to explore the mathematical object from every angle and level of detail.

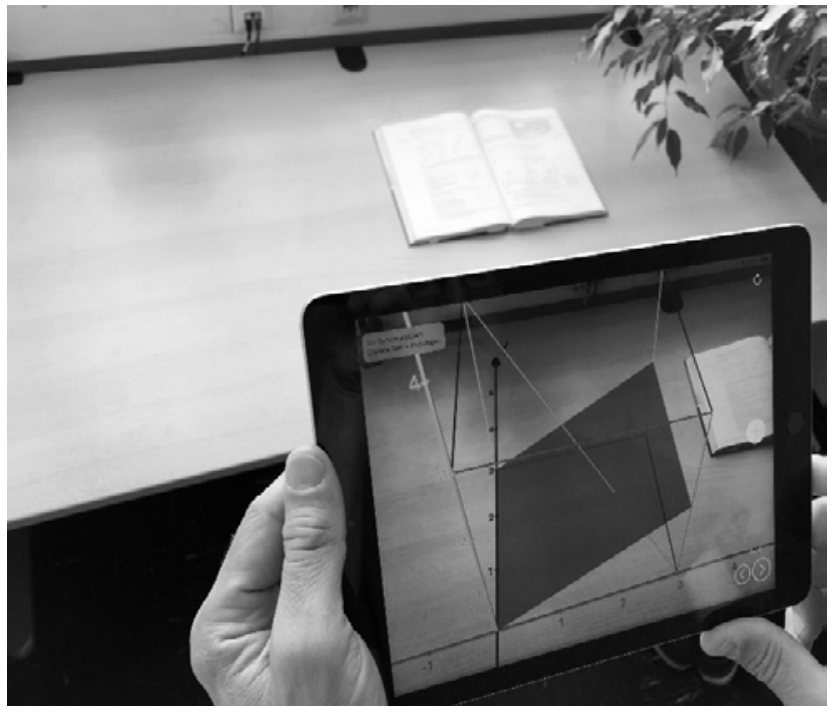

Figure 1. AR-App "GeometAR" (source D. Birnbaum): View thorugh the iPad-camera showing the reality (desk) augmented with a cube posititioned within a fixed coordinate system, a plane and straight line.

In literature there is no consistent definition of AR. The definitions range from a narrower characterisation highlighting technological aspects, to broader views on AR. In accordance with Wu et al. (2013, p. 42) a broader definition which include the application of different devices (desktop PCs, tablets, smartphones, data goggles, ...) seems to better portray the necessities in the field of education. AR can be understood as situation in which real world content is dynamically overlaid with related location- or content-specific information (Klopfer \& Squire, 2008, p. 205). This perception emphasizes that AR is technology-based but also needs to be conceptualised to enrich the situation. Thus, AR should be understood as a concept rather than a technology to bring its education-theoretical aspects to the fore (Zhou, Duh, \& Billinghurst, 2008).

\section{Added value of AR}

The benefits of AR for the teaching and learning in the field of mathematics instruction are based on

- $\quad$ organisational aspects, which are especially important for teachers, 
- physical aspects based on the theory of embodiment and the cognitive load theory

- and cognitive aspects, which characterize the symbolic relation between physical objects and abstract mathematical concepts.

\subsection{Organisational aspects}

Organizational aspects often complicate the integration of digital media in class. Problems range from changing class room to PC room, time-consuming login procedures and long learning phases for special PC programs, to name but a few. The recently developed smartphone compatibility of AR gives teachers more organisational scope and gives learners the possibility to learn in a familiar setting. The operation through body movement as intuitive pattern of action is a decisive advantage of AR. The implementation of virtual objects in the immediate real environment of the learners facilitates the understanding of the situation.

\subsection{Physical aspects}

The theory of embodied cognition describes the mutual influence between physical interaction and human thinking (Tran, Smith, \& Buschkuehl, 2017). It is closely related to the cognitive load theory (cf. Sweller, 1988) which assumes that learning results in cognitive load and describes how learning can be facilitated or complicated. Physical body movements, as they are necessary to change the perspective using AR, can support the understanding of spatial coherences (Bujak, et al., 2013, p. 538). Instead of imagining the mathematical situation, learners can enactively explore it such that there are larger cognitive capacities to understand mathematical correlations and solve the task.

\subsection{Cognitive aspects}

Mathematical concepts and its physical representations are linked by symbolic language. The possibility of spatiotemporal contiguity of physical and symbolic representation through AR can help learners to see correlations and to better understand underlying mathematical concepts (Bujak, et al., 2013, p. 540). Especially in upper secondary the mathematical concepts get more abstract and, thus, more demanding. The direct spatiotemporal representation of symbolic language (e.g. symbolic representation of lines and planes) together with their enactive-iconic (via AR) representation, can facilitate the coherence for learners by combining originally disjoint representations.

\section{AR in analytic geometry: the project MalAR}

Besides the use of AR as additional source of information, its application in mathematics in the subject area of geometry is obvious. While physical representations can be used in lower secondary education, it becomes difficult beyond, particularly in analytic and differential 
geometry because of the increasing complexity of mathematics representations. Those topics require a high level of spatial ability which can be visually supported by e.g. dynamic geometry software or AR. AR offers additional promising opportunities mentioned in section 3 , whose effects should be subject of research.

The aim of MalAR (Eng. transl.: learning mathematics with AR - an empirical study about the learning effectiveness of AR in analytic geometry) is the development and testing of an AR learning environment, which supports learning of analytic geometry by enactively operating within the learning environment. An AR-App provides mathematics tasks which correspond to the mathematics class. This AR-representation enables learners to explore the mathematics situation independently by moving within the situation. According to the intended added value of AR described in section 3, questions about the learning effectiveness and impact on spatial ability can be deduced. MalAR aims at placing the AR learning environment on a learning theory footing to provide substantiated and effective applications. MalAR pursues the following key questions:

1. What kind of effect does the AR-learning environment have in terms of learning effectiveness compared to conventional instruction?

2. What kind of effect does the AR-learning environment have in terms of spatial ability compared to conventional instruction?

Key question 1 will be investigated by a quasi-experimental intervention study in the subject area "planes". The performance of experimental groups will be compared to control groups who do not use the AR learning environment in mathematics class. Key question 2 addresses the spatial ability which is especially important in analytic geometry. By investigating correlations of key question 1 and 2, relevant factors of the AR learning environment on learning may be evaluated in more detail.

\subsection{The AR learning environment}

The AR learning environment is developed for the subject area "planes" within the wider topic analytic geometry for upper secondary mathematics classes. Especially distance calculation and position considerations are of interest. In conventional mathematics class a visualisation of the mathematical situation is limited to oblique projection.

During the topic "planes" the learners of the experimental groups elaborate on tasks which are additionally implemented in the AR-app. Through the AR-representation of the mathematics situation learners can view and explore the situation by moving their body around it. Furthermore, learners can type in their solution and visually investigate its correctness. In contrast to conventional mathematics class the AR learning environment allows for an enactive involvement in the mathematics situation. The following example demonstrates the AR-app. 
A plane $E_{1}: 2 x-y-z=-1$ is given. We are looking for a plane $E_{2}$, which contains point $A(3|1| 2)$ and is orthogonal to plane $E_{1}$. Determine $E_{2}$ and the intersecting line $g$ of the two planes.

The AR-App visualizes the given information “walk-on-able” within the smartphone's camera image by virtually adding a three-dimensional fixed coordinate system. An input area is provided to type in $E_{1}$ and $A$ (Figure 3 left). After having calculated the solution by hand, the learner can type in his solution (Figure 2, Figure 3 right) and visually check its correctness by exploring the situation from different views changing the camera perspective through body movement.

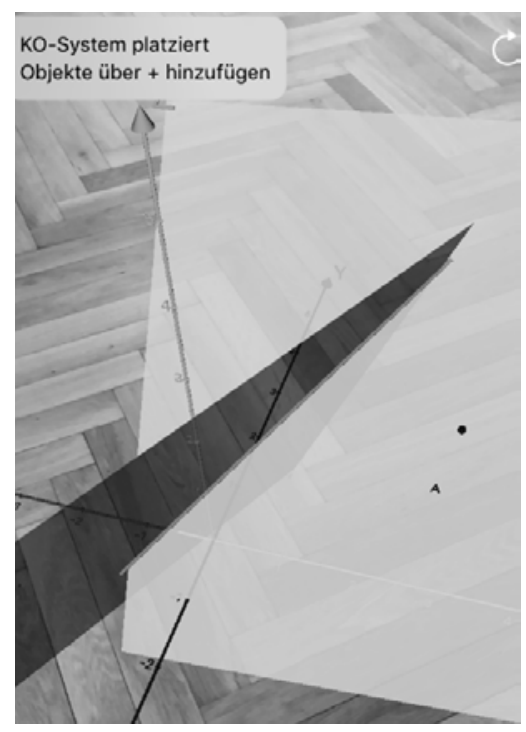

Figure 2. Screenshot of the AR-App representation of the solution. $E_{1}$ (dark grey), $E_{2}$ (pale), intersecting line $g$ and point A. 


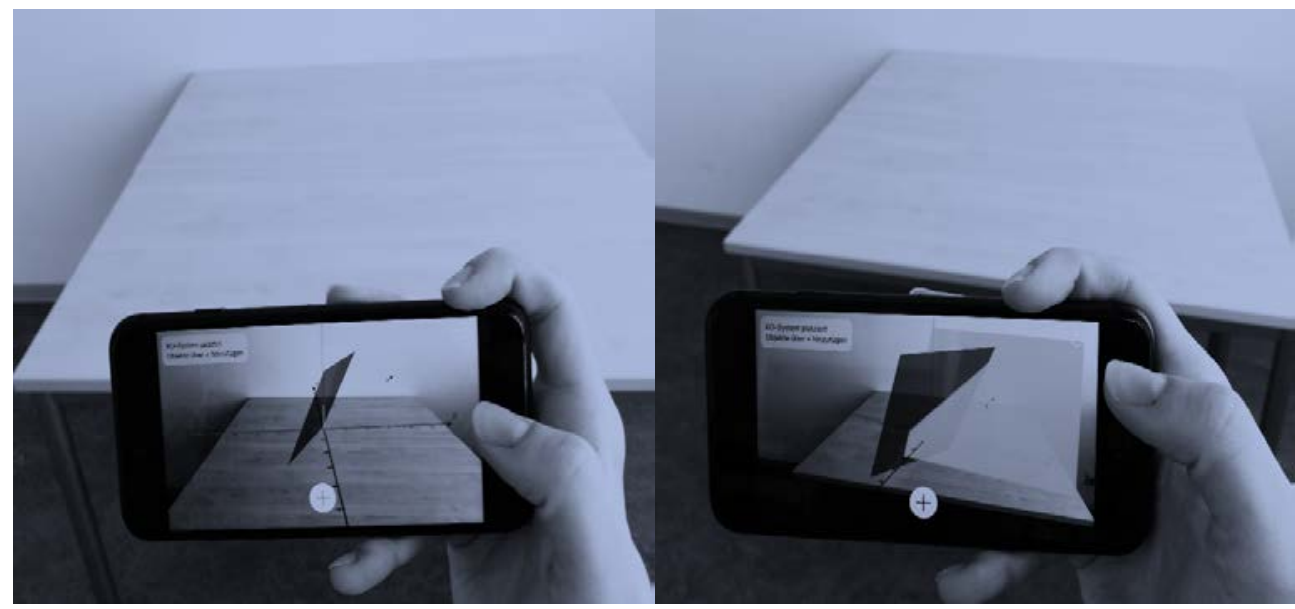

Figure 3. Visualisation of coordinate system, plane $E_{1}$ and $A$ (left). The solution, plane and intersecting lineg (right)

\subsection{Method}

During a pilot phase the further development and testing of the AR-app and AR learning environment is of primary importance. The AR-app is based on the unpublished iOS-app "GeometAR” (Birnbaum \& Ludwig, 2017) in cooperation with the working group MATIS 1from the Goethe-University Frankfurt.

During a quasi-experimental study with experimental and control groups key questions 1 and 2 are particularly in focus. The mathematics class of the experimental groups is supported by the AR learning environment. Prior to the treatment both groups sit a mathematics test to factor in mathematics achievement when considering key question 1.

Following the treatment, all learners sit a post-test consisting of former Abitur assignments. With the results of the post-test it can be investigates if the AR learning environment has effects on the learning in the subject area "planes". With reference to key question 2 the learners also sit a test concerning spatial ability to determine effects of the treatment on this specific content.

\section{Outlook}

The integration of digital everyday technology, like smartphones, has still not found its way into many schools in Germany. Not least missing teaching concepts contribute to this situation. In the area of AR, as the newest development in this area, most of the studies consider implementation opportunities without investigating the learning theory background. Besides the many known chances of AR in general it is worth looking at the teaching situation. The perspective can be shifted by the AR learning environment from a teacher- 
centred to a more learner-centred one where the learner's mathematical actual involvement can be increased (cf. Schratz, 2009). With the findings and results of the study evidencebased didactical insights in the teaching and learning with AR in mathematics instruction can be identified and needs for future AR-supported learning scenarios will can be revealed.

A next step is the extension of the AR learning environment to further subject areas. Furthermore, the AR-App will be equipped with a self-implemented module where teachers can implement tasks by themselves which they then want to use in their class.

\section{References}

Avanitis, T. N., Petrou, A., Knight, J. F., Savas, S., \& Gargalakos, M. (2007). Human factors and qualitative pedagogical evaluation of a mobile augmented reality system for science education used by learners with physical disabilities. Personal and Ubiquitous Computing, 13(3), pp. 243-250.

Azuma, R. T. (1997). A survey of augmented reality. Presence-Teleoperators and Virtual Environments 6(4), pp. 355-385.

Birnbaum, D., \& Ludwig, M. (2017). Augmented Reality im Mathematikunterricht. Ein Überblick derzeitiger Einsatzmöglichkeiten. Poster auf der 53. Jahrestagung der GdM. Potsdam.

Bujak, K. R., Radu, I., Catrambone, R., MacIntyre, B., Zheng, R., \& Golubski, G. (2013). A psychological perspective on augmented reality in the mathematics classroom. Computers \& Education 68, pp. 536-544.

Johnson, L., Levine, A., Smith, R. S., \& Haywood, K. (2010a). Key emerging technologies for elementary and secondary education. Education Digest, 76(1), pp. 36-40.

Johnson, L., Levine, A., Smith, R. S., \& Haywood, K. (2010b). Key emerging technologies for postsecondary education. Education Digest, 76(2), pp. 34-38.

Kaufmann, H., \& Schmalstieg, D. (2003). Mathematics And Geometry Education With Collaborative Augmented Reality. Computers \& Graphics, 27(3), pp. 339-345.

Kaufmann, H., Steinbügl, K., Dünser, A., \& Glück, J. (2005). General Training of Spatial Abilities. In Annual Review of CyberTherapy and Telemedicine: A Decade of VR 3 (pp. 65-76).

Kerawalla, L., Luckin, R., Seljeflot, S., \& Woolard, A. (2006). "Making it real": exploring the potential of augmented reality for teaching primary school science. Virtual Reality, (10)3, pp. 163-174.

Klopfer, E., \& Squire, K. (2008). Environmental detectives: the development of an augmented reality platform for environmental simulations. Educational Technology Research and Development, 56(2), pp. 203-228.

Martin, S., Diaz, G., Sancristobal, E., Gil, R., Castro, M., \& Peire, J. (2011). New technology trends in education: seven years of forecasts and convergence. Computers \& Education, 57(3), pp. 1893-1906. 
Martin-Gutierrez, J., Saorin, J. L., Contero, M., Alcaniz, M., \& Perez-Lopez, D. C. (2010). Design and validation of an augmented book for spatial abilities development in engineering students. Computers \& Graphics 34(1), pp. 77-91.

Milgram, P., \& Kishino, F. (1994). A taxonomy of mixed reality visual displays. IEICE Transactions on Information Systems, 77(12), E77-D(12).

Schratz, M. (2009). ,Lernseits’ von Unterricht. Alte Muster, neue Lebenswelten -was für Schulen? Lernende Schule 12(46-47), pp. 16-21.

Sweller, J. (1988). Cognitive load during problem solving: Effects on learning. Cognitive Science, 12 (2), pp. 257-285.

Tran, C., Smith, B., \& Buschkuehl, M. (2017). Support of mathematical thinking through embodied cognition: Nondigital and digital approaches. Cognitive Research: Principles and Implications 2:16.

Wu, H.-K., Lee, S. W.-Y., Chang, H.-Y., \& Jyh-Chong, L. (2013). Current status, opportunities and challenges of augmented reality in education. Computers \& Education 62, pp. 41-49.

Zhou, F., Duh, H.-B.-L., \& Billinghurst, M. (2008). Trends in augmented reality tracking, interaction and display: A review of ten years of ISMAR. In Proceedings of the 7th IEEE/ACM International Symposium on Mixed and Augmented Reality. Cambridge, United Kingdom. 